\title{
Testing a Novel Method for Initializing Air Parcel Back Trajectories in Precipitating Clouds Using Reanalysis Data $\mathscr{O}$
}

\author{
ANNIE L. PUTMAN \\ Department of Geology and Geophysics, University of Utah, Salt Lake City, Utah \\ Xiahong Feng and ERIC S. Posmentier \\ Department of Earth Sciences, Dartmouth College, Hanover, New Hampshire \\ ANTHONY M. FAIIA \\ Department of Earth and Planetary Sciences, University of Tennessee, Knoxville, Tennessee \\ LESLIE J. SONDER \\ Department of Earth Sciences, Dartmouth College, Hanover, New Hampshire
}

(Manuscript received 20 March 2017, in final form 18 September 2017)

\begin{abstract}
Lagrangian air parcel tracking is a powerful tool for estimating vapor source locations, particularly for isotope hydrology applications. Identified vapor source regions may be sensitive to the distribution of altitudes at which back trajectories are initiated. Ideally, those initial altitudes should reflect the altitudes where precipitation forms. This paper introduces a novel method for estimating these heights from reanalysis data and an air parcel lofting routine, which is referred to as the "Reanalysis" method. Using Barrow, Alaska (now known as Utqiagivik), as a test site, the study compares the distribution of air parcel initiation heights and vapor source conditions from back trajectories initiated at 1) heights determined by the Reanalysis method and 2) heights acquired from $35-\mathrm{GHz}$ vertically resolved cloud radar, termed the "Cloud Radar" method. Only 2 of the 70 events failed to produce condensation at any elevation. The distribution of air parcels generated by each method was compared on the basis of the median height and the median-adjusted overlap, and yielded excellent $(n=28)$, good $(n=20)$, fair $(n=6)$, and poor $(n=14)$ matches between the two methods. The excellent and good category events tended to produce condensation profiles with lower median heights, which translated to more similar vapor source characteristics. Poorly matched events tended to result from rain events where the Reanalysis method yielded much higher median heights than the Cloud Radar method.
\end{abstract}

\section{Introduction}

There has been increasing interest in investigating the sources and transport of water vapor (Sodemann et al. 2008; Pfahl and Wernli 2008; Gimeno et al. 2012; Bailey et al. 2015) using Lagrangian back trajectory methods. In the context of hydrologic cycling, such studies allow for identifying vapor source regions and for studying the

Supplemental information related to this paper is available at the Journals Online website: https://doi.org/10.1175/ JTECH-D-17-0053.s1.

Corresponding author: Annie Putman, putmanannie@gmail.com variation in water vapor transport patterns over a variety of temporal scales (e.g., storm, seasonal, and interannual). This information, used independently or in conjunction with studies of the stable isotopes of precipitation, is important for understanding the response of the hydrologic cycle to changes in atmospheric circulation and thermal structure.

In back trajectory methods, the location over ocean or land where the water vapor originates is termed the "vapor source" or "evaporation site," and various specified elevations above the precipitation site are the "arrival heights." To find evaporation sites for precipitation, the back trajectory method tracks air parcels backward in time, guided by reanalysis winds, beginning at the arrival 
heights above the precipitation site. In our study the back trajectory ends at the time and locality at which the parcel initially lifted from the planetary boundary layer (PBL) into the free troposphere. The PBL is an appropriate indicator of the vapor source region for precipitation isotope studies, because as a result of the dominance of vertical mixing, the vapor content of the air parcel is rapidly and directly affected by the sea surface. We ignore the possibility that air parcels may move in and out of the boundary layer multiple times (e.g., Sodemann et al. 2008), although that consideration may be necessary for a detailed water balance study.

Back trajectories provide a powerful tool for linking observed precipitation to the vapor source region as long as the analysis meets three requirements: 1 ) there is little entrainment of vapor into or out of an air parcel after its final departure from the PBL, 2) reanalysis data reliably describe actual meteorological conditions, and 3) appropriate altitudes are selected to begin back trajectories. This article tests the sensitivity of a new method for addressing the third requirement.

Finding the proper altitude to initiate a back trajectory is important for at least two reasons. First, wind direction and velocity change with altitude, and therefore air parcels released at different heights may indicate very different vapor sources. Any inaccuracy in starting altitudes propagates to inaccurately identified source regions. Second, the atmospheric conditions (temperature, pressure, and humidity) associated with condensation or deposition are specific to the altitude of condensation or deposition. These conditions can be used for determining the amount of the original vapor remaining in the air parcel when it arrives at the precipitation site, and for determining the type and properties of the precipitation. For example, investigators interested in understanding isotopic ratios of precipitation will want to know the temperature of condensation/deposition (for equilibrium fractionation) or humidity at the cloud base (to assess the potential for subcloud evaporation). We expect that the vapor sources identified by back trajectories are sensitive to the choice of arrival heights, and so are the conclusions (e.g., meteorological conditions at the source) drawn from the analysis.

Within the literature, there are many ways of selecting air parcel arrival height. Methods vary from a single back trajectory per event with a fixed arrival height to many trajectories at fixed pressure intervals. For example, Theakstone (2011) tracked a single air parcel starting at $500-\mathrm{m}$ altitude above Tustervatn, Norway, backward in time to its source region every $24 \mathrm{~h}$ for 7 years. In a similar study (Reijmer and Broeke 2001), trajectories arriving at six different fixed heights above Dronning Maud Land, Antarctica, were backtracked every $12 \mathrm{~h}$ for 1 year. Most multiyear precipitation stable isotope studies estimate the vapor source using relatively few starting heights in order to balance computation time with the large number of trajectories required for long-term datasets (Breitenbach et al. 2010; Kurita 2013). Although tracking relatively few parcels is simple, it risks misidentification of the actual vapor source. Precipitation may occur over a range of heights, and heights may vary considerably among precipitation events at a single site or even during a single event. Furthermore, using just one or a few trajectories may not provide adequate statistical sampling to identify the vapor source region accurately.

Another method tracks all air above the precipitation site by discretizing the air column into equalmass air parcels at fixed intervals of atmospheric pressure and sending air parcels from multiple grid points at fixed time intervals (Stohl and James 2004, 2005). This method is thorough and allows for sophisticated postprocessing of trajectories and a detailed water budget analysis for a range of spatial scales. Because it does not require any prior knowledge of precipitation location and timing, it is also very flexible. However, not all trajectories tracked contribute vapor equally to the precipitation. This means that postprocessing would be required to calculate contributions of vapor, and thus the relative weighting to be applied to source and along-trajectory weather variables. Additionally, the computational expense of tracking large numbers of trajectories over large regions may be prohibitive for researchers with limited access to processor speed and time.

By incorporating meteorological and precipitation record information, it is possible to retain the simplicity and efficiency of using a small number of back trajectories yet have the representation of whole air column tracking. For example, in a study of vapor source shifts for central Greenland (Sodemann et al. 2008), based on the premise that higher relative humidities signaled a greater likelihood of condensation, air parcels above central Greenland were tracked only if their relative humidity exceeded $80 \%$. Our approach uses reanalysis estimates of relative humidities and vertical velocities in the air column above the precipitation site to predict condensation rates. In addition, we divide the condensing/depositing portion of the air column into discrete air parcels such that each air parcel represents an equal mass of precipitate. Our method yields a more realistic sampling of the vapor source than methods that backtrack 10 or fewer air parcels. Compared with a comprehensive air column tracking method, our method minimizes the computation time required to run back trajectories and the postprocessing time to extract the 
subset of trajectories relevant to an event, with no loss in useful information.

Partitioning the air column into discrete air parcels containing equal amounts of water allows vapor source conditions to be easily averaged and evaluated (Putman et al. 2017). Furthermore, the source region may be divided into subsets based on sea surface temperature (Everhart 2011) or other variables to assess the relative importance of different regions contributing to a precipitation event.

The efficacy of our approach depends on an accurate determination of condensation/deposition rate as a function of altitude. We refer to this as a "condensation rate profile," although the profile may represent either condensation and/or deposition of vapor onto ice, depending on the cloud conditions. We consider the amount of precipitation from a given altitude to be proportional to the condensation rate of that altitude and that the proportionality is the same for all heights. Though this is an assumption, it is reasonable because we average over multihour time scales in fully developed precipitating clouds. Thus, both the retention of condensate in the cloud and the rate of reevaporation below clouds are likely small.

The condensation rate profile can be determined from millimeter-wavelength cloud radar (MMCR) by using the inferred precipitation rate as a function of altitude. Algorithms to convert the Doppler cloud radar returns to precipitation rate already exist (Zhao and Garrett 2008). Furthermore, because the radar directly observes the precipitating cloud with high vertical resolution, the condensation rate profile produced is very accurate. This method for determining the condensation rate profile is referred to as the "Cloud Radar method," and it would be the primary method for determining the condensation rate profile except that millimeter-wavelength cloud radar deployments are uncommon and relatively recent, with data collection only as far back as the 1980s. However, the number of cloud radars is increasing due to the DOE ARM (Kollias et al. 2007) and European Cloudnet (Illingworth et al. 2007) deployments. Because of the limited availability of data for this method, a more universally applicable method for determining the condensation rate profile is desirable.

We introduce a method called the "Reanalysis method," which incorporates reanalysis data into a lofting model to identify altitudes where condensation and/or deposition is occurring. This method is based on the proportionality of condensation rate to vertical velocity and air density, and the vertical gradient of specific humidity. All of these variables are available directly or indirectly from reanalysis data. Because reanalysis data are available globally and back in time for decades, the
Reanalysis method for identifying vapor sources of precipitation events is broadly applicable. However, to apply the method with confidence, a thorough evaluation of the reliability of the method is required.

The Cloud Radar method condensation profile and the Reanalysis method condensation profile are not expected to match perfectly. This is because the former is from high-resolution data at a single site and the latter results from the average conditions in the GDAS pixel that includes the site. However, the comparison is useful because Lagrangian back trajectories using gridded data are typically used to evaluate vapor sources for a particular site. Thus, we compare the results of the Cloud Radar and Reanalysis methods to assess the accuracy and applicability of the Reanalysis method so that it may be used with confidence at times and locations when cloud radar is unavailable. Once verified, the Reanalysis method will be appropriate for researchers working on relatively fast laptop or desktop computers. Because accurate identification of the source region is important for accurate estimates of evaporation conditions and of the temperature gradient from source to condensation site, we expect the Reanalysis method should be of particular interest to those who use back trajectories to interpret stable isotopic variations in precipitation samples. Likewise, other studies (e.g., Keegan et al. 2014; Fig. S4) using back trajectories can benefit from the accessibility and computational ease of our methods.

The following sections describe the Lagrangian method used (section 2a), and detail the Reanalysis (section 2b) and Cloud Radar (section 2c) methods. The results presented assess the accuracy of the condensation rate vertical profile calculated using the Reanalysis method by comparison with the condensation rate profile determined from Cloud Radar data for 70 storm events between January 2009 and April 2013 at the Department of Energy (DOE) Atmospheric Radiation Measurement (ARM) site at Barrow, Alaska [now known as Utqiagivik; North Slope of Alaska (NSA), $71.32326^{\circ} \mathrm{N}, 156.6157^{\circ} \mathrm{W}$ ]. To contextualize the importance of the back trajectory initialization heights, we investigate the sensitivity of the estimated vapor source region to the condensation rate profile.

\section{Methods}

\section{a. HYSPLIT and tracking procedure}

This work uses the Hybrid Single-Particle Lagrangian Integrated Trajectory model (HYSPLIT) (Draxler 1999; Draxler and Hess 1997, 1998). HYSPLIT uses reanalysis data from the Global Data Assimilation System (GDAS) to trace the trajectories and to identify the 
origins of air parcels. GDAS data are reported with 3-h temporal resolution and $1^{\circ} \times 1^{\circ}$ spatial resolution at 24 irregularly spaced pressure heights, with higher resolution near Earth's surface.

To begin a HYSPLIT back trajectory run, we first identified the time of inception of the back trajectories. Because the reanalysis files used for tracing the back trajectories had 3-h resolution, a representative 3-h window was selected for each storm event. The ARM NSA precipitation sampling record provided the precipitation start and end times that constrained the potential 3-h windows. The surface analysis maps of Alaska provided by the National Centers for Environmental Prediction, available at 3-h intervals, showed when precipitation was reported and its intensity. The cloud radar was examined for signal returns associated with precipitation (Bharadwaj et al. 2011; Johnson and Jensen 1996). Within the period specified by the precipitation event record, a 3-h interval with the most intense radar reflectivity and Doppler vertical velocity returns was selected, with preference for periods where precipitation occurred for the duration of the 3-h interval. The period selection was confirmed by referencing the surface analysis maps.

Second, we identified air parcel arrival heights above the precipitation site based on where our methods indicated condensation or deposition onto snow or ice occurred. This method assumes that vertical mixing at the precipitation site is weak and so is appropriate for the meteorology at Barrow (Curry 1986; Shupe et al. 2008). Our methods for calculating the condensation rate profiles are described in sections $2 b$ and $2 c$ for the Reanalysis and Cloud Radar methods, respectively. The condensation rate profiles were subdivided vertically into 1000 vapor-containing air parcels, distributed in proportion to the rate of condensation, such that each parcel contained an equal mass of water.

Once the vertical distribution of air parcels was determined, HYSPLIT tracked the parcels from the arrival heights backward in time for 10 days $(240 \mathrm{~h})$ to the evaporation site. HYSPLIT calculated air parcel movement by advection based on the wind velocity data from the GDAS reanalysis.

The vapor source was identified as the location where the trajectory indicated that the air parcel first crossed into the PBL, that is, where the original forward trajectory ascended from the PBL into the free atmosphere. The PBL height used was that reported in the GDAS reanalysis. This criterion for assigning a vapor source assumes strong mixing within the PBL and, compared with the method of Sodemann et al. (2008), who track the specific humidity changes of an air parcel as it enters and leaves the PBL, may slightly bias vapor sources proximal to the sampling site. However, this method should give a statistically robust estimate of the vapor source locations and conditions. At the vapor source, the sea surface temperature $V_{\text {SST }}$ (NOAA 2013), relative humidity with respect to the sea surface temperature $V_{\mathrm{hSST}}$, and hydrogen isotopic composition of the vapor at the source $\delta^{2} H_{v}($ Good et al. 2015) were tabulated.

\section{b. The Reanalysis method}

The Reanalysis method of producing the condensation profile is conceptually similar to methods presented in O'Gorman and Schneider (2009). Other methods for identifying the altitudes of moisture contributing to precipitation include using the relative humidity profile (Sodemann et al. 2008), the specific humidity profile, or cloud and ice variables from reanalysis. Cloud and/or ice variables were not available in the GDAS reanalysis, and the cloud and/or ice variable values reported in other reanalysis products may result from a combination of hydrometeor production and vertical transport due to throughfall or vertical mixing; therefore, they may not reflect the elevations and concentrations of the source moisture. The humidity profile (relative or specific) may be a useful simplification in cases where the meteorology indicates precipitation results from deep convection.

Temperature $T$, relative humidity $\mathrm{RH}$, and vertical velocity $W$, of the air at systematic pressure heights $P$, are reported as part of the GDAS reanalysis. We used these data from the pixel covering $71^{\circ}-72^{\circ} \mathrm{N}, 156^{\circ}-$ $157^{\circ} \mathrm{W}$ that includes Barrow to calculate a condensation rate profile.

The condensation rate $C\left(\mathrm{~g} \mathrm{~m}^{-3} \mathrm{~s}^{-1}\right)$, based on mass and energy conservation of an idealized air parcel that experiences condensation upon moist adiabatic uplift, is

$$
C=-\frac{\delta Q}{\delta P} W \rho
$$

In Eq. (1), $Q$ is specific humidity ( $\mathrm{g} \mathrm{kg}^{-1}$ moist air), $P$ is pressure $(\mathrm{hPa}), W$ is vertical velocity $\left(\mathrm{hPa} \mathrm{s}^{-1}\right)$, and $\rho\left(\mathrm{kg} \mathrm{m}^{-3}\right)$ is the density of the moist air. The density of moist air is calculated based the ideal gas law, where $\rho=P M_{\text {air }} / T R, T$ is temperature, $R$ is the gas constant, and $M_{\text {air }}$ is the molecular weight of moist air.

Change to $Q$ is caused by condensation. The rate of change in specific humidity with respect to pressure, $\delta Q / \delta P$, is approximated by the difference ratio of $\Delta Q$ to $\Delta P$ from an upward finite-difference operator with a fixed $10-\mathrm{hPa}$ decompression difference, that is, $\Delta P=$ $-10 \mathrm{hPa}$. The specific humidity $Q$, before adiabatic decompression, is calculated from $\mathrm{RH}$ and saturation specific humidity $Q_{\text {sat }}$. If $\mathrm{RH}$ is less than $100 \%$, then calculated $Q$ will be less than its saturation value $Q_{\text {sat }}$. 
During adiabatic uplift, if vapor in the air parcel does not reach saturation, then $Q$ will not change with the change in pressure (i.e., $\Delta Q=0$ and thus $C=0$ ), and the final temperature will be entirely due to dry adiabatic decompression (with a lapse rate of $9.8^{\circ} \mathrm{C} \mathrm{km}^{-1}$ ). However, if during uplift the air parcel reaches saturation and condensation occurs, then $Q$ will decrease; the latent heat of condensation will heat the air parcel and thus the extent of cooling will be less than that due to dry adiabatic decompression alone. In such a moist adiabatic process, the final state of the air parcel undergoing a $10-\mathrm{hPa}$ pressure decrease will depend on the following three relationships: 1) mass conservation (the change in $Q$ equals condensate amount),2) energy conservation (latent heat released by condensation equals the increase in internal energy associated with temperature change, minus the work done by expansion), and 3) the relationship between $Q_{\text {sat }}$, temperature, and pressure. Computationally, $\delta Q / \delta P$ is solved iteratively, looping through the three relationships, and the converged solution is then used to calculate $C$.

Because clouds forming in the Arctic are typically mixed-phase clouds, $Q_{\text {sat }}$ used in the Reanalysis method results from

$$
Q_{\text {sat }}=Q_{\text {sat,liq }} f_{\text {liq }}+Q_{\text {sat,ice }}\left(1-f_{\text {liq }}\right),
$$

where $Q_{\text {sat,liq }}$ and $Q_{\text {sat,ice }}$ are the temperature-dependent saturation vapor pressure values for liquid and ice from equations in Alduchov and Eskridge (1996). Using a fraction $f_{\text {liq }}$ to estimate the bulk effects of mixed-phase clouds is consistent with methods presented in Nusbaumer et al. (2017) for the Community Atmosphere Model. The fraction of liquid $f_{\text {liq }}$ comes from

$$
f_{\text {liq }}=\frac{1+\operatorname{erf}\left(\frac{T+10}{4}\right)}{2}(\text { Code S1, lines } 43 \text { and } 73) \text {, }
$$

where $T$ is the temperature $\left({ }^{\circ} \mathrm{C}\right)$ of the air parcel.

Equation (1) is applied at each of the pressure heights reported by the GDAS reanalysis and is converted to an hourly condensation rate. The discrete values of $C$ at the pressure heights are converted to a continuous condensation rate profile by linear interpolation. The resulting condensation rate profile is used to determine the altitudes at which the 1000 air parcels begin their back trajectories. A MATLAB subroutine used for this method is included in the supplemental materials (Code S1).

\section{c. The Cloud Radar method}

The Cloud Radar method of estimating condensation rate as a function of altitude has been tested and verified by Zhao and Garrett (2008). We used this method without modification, so we include only a brief description of the method here to provide a basis for comparison with the newly developed, experimental Reanalysis method.

Barrow hosted two similar vertically pointing Doppler cloud radars over the period of this study: an MMCR (Johnson and Jensen 1996) from January 2009 to April 2011 and a Ka-band zenith radar (KAZR; Bharadwaj et al. 2011) after April 2011. Both instruments operated at a frequency of approximately $35 \mathrm{GHz}$ and recorded the first three Doppler moments: reflectivity $Z$, Doppler vertical velocity $V_{\mathrm{Dm}}$, and spectral width. The instruments operated in four modes: general, precipitation, boundary layer, and cirrus. The strengths of the four MMCR modes were combined into the active remotely sensed clouds locations (ARSCL; Clothiaux et al. 2000) value-added product and were available at 75-m vertical resolution between ground level and $20 \mathrm{~km}$, every $10 \mathrm{~s}$. The more sensitive KAZR data were available at $30-\mathrm{m}$ resolution between ground level and $20 \mathrm{~km}$ in cross- and copolarized modes at less than 10-s intervals. To maintain the comparability of results before and after the instrumentation switch, only copolarized data from the new instrument were used. No product similar to ARSCL was available for the KAZR, so data recorded in general mode were used. Although no correction was performed to reconcile the vertical resolution or receiver sensitivity difference, no systematic difference between the results from the two instruments was detected.

Reflectivity $Z$ and Doppler vertical velocity $V_{\text {Dm }}$ were processed using algorithms developed by Zhao and Garrett (2008) to produce a vertically resolved precipitation rate $\mathrm{Pc}\left(\mathrm{g} \mathrm{m}^{-2} \mathrm{~s}^{-1}\right)$ that was differentiated with respect to altitude to produce $C\left(\mathrm{~g} \mathrm{~m}^{-3} \mathrm{~s}^{-1}\right)$. The calculations were performed at discrete altitudes $i$ above Barrow where the Doppler signals $V_{\mathrm{Dm}}$ and $Z$ are available. Precipitation rates for snow and ice are calculated in terms of the rain-rate equivalent.

The precipitation rate at altitude $i, \mathrm{Pc}_{i}\left(\mathrm{~g} \mathrm{~m}^{-2} \mathrm{~s}^{-1}\right)$, can be obtained from the hydrometeor volume $\left(4 / 3 \pi r_{i}^{3}\right)$, number density $N_{i}$, and hydrometeor bulk density relative to liquid $\rho_{p} / \rho_{l}$ :

$$
\mathrm{Pc}_{i}=\frac{4 / 3 \pi\left(r_{i}^{3}\right) \rho_{p}\left(V_{\mathrm{Dm}, i}\right)\left(N_{i}\right)}{\rho_{l}} .
$$

The hydrometeor radius $\left(r_{i}\right)$ used in Eq. (3) is calculated as

$$
r_{i}=A\left(T_{i}\right) V_{\mathrm{Dm}, i}^{B\left(T_{i}\right)}
$$




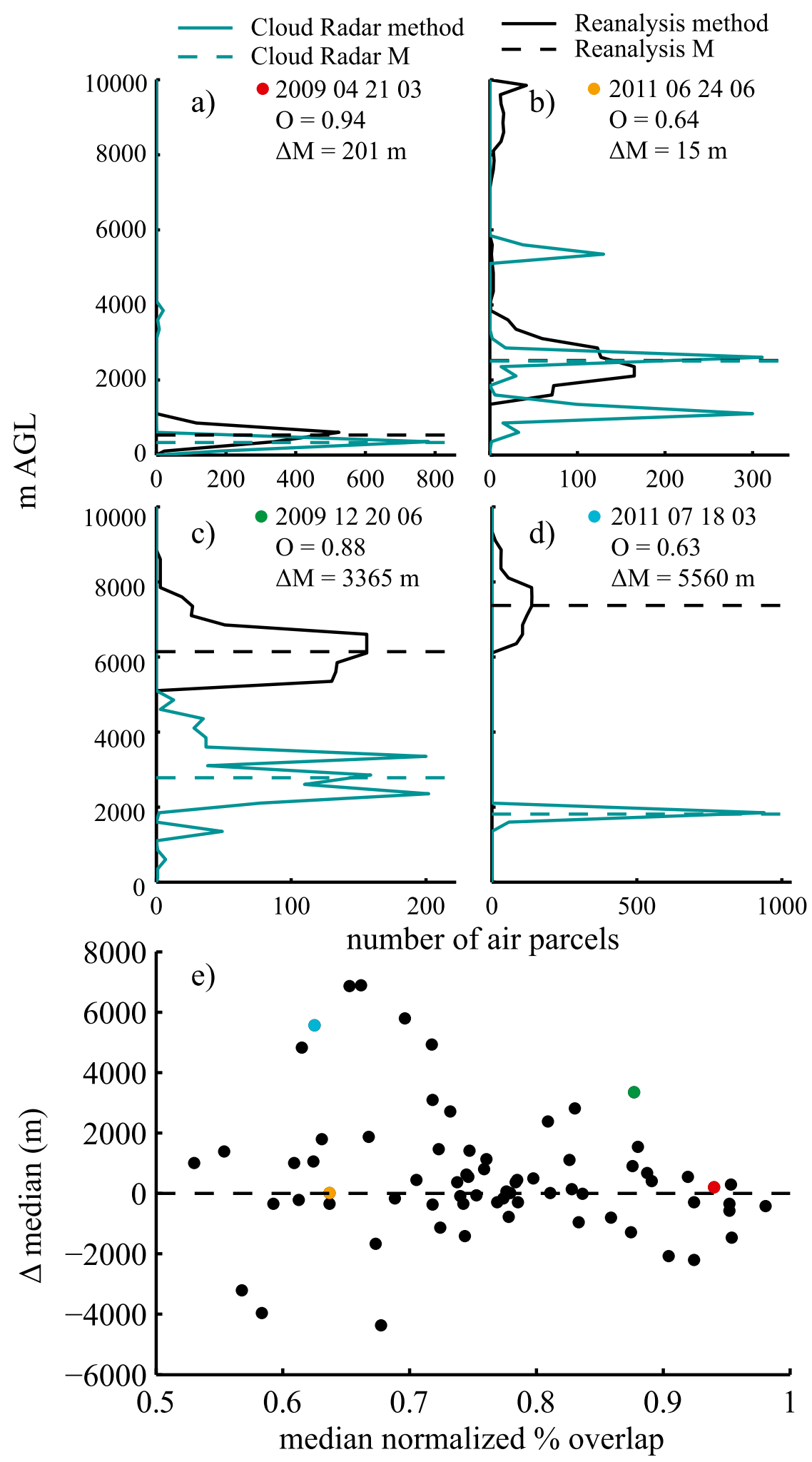

FIG. 1. Difference between the methods' median $M$ and $M$-normalized overlap $O$ is presented with examples of four condensation profile scenarios. (a)-(d) The $y$ axis is the elevation (MSL) and the $x$ axis is the number of air parcels in a 250-m bin. (a) Excellent match, low $\Delta M$, high $O$. This indicates air parcel distributions with similar midpoints and similar 
with coefficients $A\left(T_{i}\right)$ and $B\left(T_{i}\right)$ accounting for hydrometeor phase and crystal habit, respectively, and to normalize snowflakes with plate or column crystal habits to their spherical raindrop equivalent. The number density $N_{i}$ is calculated as $N_{i}=Z_{i} / 2^{6} r_{i}^{6}$.

The precipitation rates $\mathrm{Pc}_{i}$ for the 3-h span were averaged to produce a single precipitation rate average $\overline{\mathrm{P}} \mathrm{c}_{i}$ for the 3-h period such that the results are comparable in temporal resolution with the results of the Reanalysis method calculation. Because decreases in $\overline{\mathrm{P}} c_{i}$ with increasing altitude correspond to condensing layers of air, while increases in $\overline{\mathrm{P}} \mathrm{c}_{i}$ with increasing altitude correspond to evaporating layers, $\overline{\mathrm{P}} \mathrm{c}_{i}$ is differentiated with respect to altitude (where $i=0$ is surface) to give the condensation rate $C_{i}\left(\mathrm{~g} \mathrm{~m}^{-3} \mathrm{~h}^{-1}\right)$ at each height:

$$
\bar{C}_{i}=\frac{-d \overline{\mathrm{P}} c_{i}}{d h_{i}}
$$

A MATLAB subroutine for this method is included in the supplemental information (Code S2).

\section{d. Evaluating the methods}

To evaluate the two methods, the condensation rate profiles were compared. To inform isotope hydrology studies, we also compared average vapor source conditions. The evaluation was performed with the understanding that comparing measurements from a point location with average conditions in a $1^{\circ} \times 1^{\circ}$ pixel will undoubtedly produce inconsistencies. Nonetheless, in the next section we evaluate how representative the gridded meteorological data are of the conditions at Barrow, and we discuss the limitations and strengths of the Reanalysis method for initiating back trajectories.

\section{1) COMPARING THE CONDENSATION PROFILES}

For each of the 70 events that produced a condensation profile, back trajectories of 1000 air parcels were initiated at a distribution of altitudes whose spacing is proportional to the rate of condensation. The condensation profiles exhibit a variety of distributions (Fig. 1), some of which are complex such that a comparison of conventional descriptive statistics like the mean and standard deviation does not adequately evaluate the differences between the methods. Instead, we evaluate the methods using three metrics-median $M$, overlap $O$, and amplitude $A$-that are conceptually similar to the structure $(S)$, amplitude $(A)$, and location $(L)$ (SAL) quality measure in Wernli et al. (2008). The latter two metrics require binning the data, and the bin width used was $250 \mathrm{~m}$.

First, the $M$ of the air parcels was calculated. The median is preferred to the mean as a measure of centrality because it is insensitive to small numbers of air parcels that are very high or very low. The absolute difference between the Reanalysis method $M$ and the Cloud Radar method $M$ is $|\Delta M|$.

Second, $O$ was calculated. This is the percent of air parcels that originate in the same 250-m bins. The overlap was calculated after normalizing the condensation profiles to their respective medians. This metric, when analyzed in tandem with $|\Delta M|$, can identify four different scenarios that occur in our data, shown in Table 1.

The $A$ of the distribution contributes to $O$, so $A$ is defined as the maximum number of parcels in any bin in the histogram. Because of the difference in vertical resolution between the Cloud Radar method and the Reanalysis method, as well as the tendency at Barrow and other Arctic locations for precipitation to occur in thin layers of the atmosphere, examining the amplitude differences may help explain some of the less favorable comparisons between the methods.

\section{2) COMPARING THE VAPOR SOURCE CONDITIONS}

Though the focus of the paper is on comparing methods for estimating condensation profiles, 1000 back trajectories were calculated for each of the 70 events, using both methods. In total, almost 140000 back trajectories were calculated. Comparing the results of the back trajectories can help assess how sensitive the estimated vapor source locations and conditions are to the back trajectory initialization heights. Because this study is focused on using back trajectories for studies of stable isotope systematics, we assessed the differences in vapor source identification by comparing $V_{\mathrm{SST}}$ (NOAA 2013), $V_{\mathrm{hSST}}$, and $\delta^{2} H_{v}$ (Good et al. 2015). Although the $\delta^{2} H_{v}$ product is a long-term monthly average, so does not capture variability among years or at time scales less than monthly, it is useful for illustrating differences at the vapor source.

distributions. (b) Good match, low $\Delta M$, low $O$. This indicates air parcel distributions with similar midpoints but different distributions. (c) Fair match, high $\Delta M$, high $O$. This indicates air parcel distributions with different midpoints but similarly shaped distributions. (d) Poor match, high $\Delta M$, low $O$. This indicates air parcel distributions that differ in midpoint and overlap. (e) All 68 events plotted in $O$ and $\Delta M$ space, with colored dots matching the examples in (a)-(d). 
TABLE 1. The four categories resulting from complementary metrics $|\Delta M|$ and $O$, and the number $n$ of events that comprise each category.

\begin{tabular}{lcccc}
\hline \hline & Excellent & Good & Fair & Poor \\
\hline$|\Delta M|(\mathrm{m})$ & $<1500$ & $<1500$ & $>1500$ & $>1500$ \\
$O$ (fractional \%) & $>0.75$ & $<0.75$ & $>0.75$ & $<0.75$ \\
$n$ (events) & 28 & 20 & 6 & 14 \\
\hline
\end{tabular}

To compare the two methods efficiently, the subset of instances of each vapor source variable associated with each event and method that were traced to within the PBL above the ocean were averaged to yield a mean, characteristic value for that event. The mean value is denoted with an overbar. On average, $416 \pm 23$ trajectories were traced to the PBL over the ocean for each event.

\section{3) Meteorological context}

We expected that the Reanalysis and the Cloud Radar methods would match best (small $|\Delta M|$, high $O$ ) for large weather systems with strong gradients in pressure and temperature spanning multiple grid cells, and match poorly for subgrid-scale weather characterized by weaker temperature and pressure gradients. Using surface analysis maps of Alaska provided by the National Centers for Environmental Prediction, storm events were categorized into synoptic, regional, and local events. If low pressure systems, fronts, and other distinct, highgradient features were associated with precipitation, then we classified the events as synoptic events. Broad troughs or weak low pressure systems that affected multiple grid cells in the region of the North Slope were classed as regional events. Events without a strong temperature or pressure gradient were classified as local.

\section{Results and discussion}

\section{a. Condensation rate profiles}

\section{1) EVENTS FOR WHICH NO CONDENSATION WAS PRODUCED}

The Reanalysis method produced a condensation rate profile of $0 \mathrm{~g} \mathrm{~m}^{-3} \mathrm{~s}^{-1}$ at all altitudes for 2 of the 70 precipitation events. The Cloud Radar method produced a nonzero condensation rate profile for all 70 events. The two events where only the Cloud Radar method produced a condensation rate profile were both categorized as local.

There are two possible reasons the Reanalysis method does not produce precipitation for the two local category events. First, the skew $T$ plots (included in the supplemental material in Media S1) and the cloud radar condensation profiles for the precipitation events indicate that precipitation typically occurred in a narrow, conditionally unstable, low-altitude layer ( $>900 \mathrm{mbar}$ ). In both cases, vapor supply was low except in a narrow band in the lower troposphere. The low-elevation thin cloud layer suggests that the mechanism for producing updrafts was cloud-top radiative cooling (Curry 1986). If the Reanalysis method captures the average conditions of the air column, then it is likely that it may not produce precipitation because it is limited by the lack of water. Second, animations from the X-band scanning ARM precipitation radar (XSAPR) deployed at the Barrow NSA site are available for one of the two missed events. The animation, available in the supplemental material (Media S1), indicates that precipitation for this event occurred over Barrow but not within the rest of the pixel. Thus, the average conditions of the $1^{\circ} \times 1^{\circ}$ pixel may not have been conducive to rain. While the Reanalysis method did not produce a condensation profile even though precipitation occurred at Barrow, the result is representative of the average conditions of the pixel. This issue of pixel average conditions differing from the conditions at the site of interest is important to consider when applying the Reanalysis method at locations without cloud radar. For example, if the precipitation typically occurs from localized convection, the method may not work well.

\section{2) COMPARING CONDENSATION RATE PROFILES}

The condensation profiles of the remaining 68 precipitation events for which both methods produced a nonzero condensation profile are compared using a combination of the three metrics- $M, O$, and $A$-and are discussed in terms of precipitation type and intensity. The average median height of the condensation profiles calculated by the Reanalysis method was $2476 \pm$ $249 \mathrm{~m}$, whereas the condensation profiles calculated by the Cloud Radar method had an average median height of $1879 \pm 163 \mathrm{~m}$. The distribution of median values for each comparison category is shown in Table 2.

When investigated together, as shown in Fig. 1 and defined in Table 1, values of $|\Delta M|$ and $O$ characterize four scenarios: excellent, good, fair, and poor matches. An example comparison between profiles is shown for each of the four scenarios. Excellent matches correspond to condensation profiles with similar midpoints and shapes. These are often a single peak. Good matches correspond to events with similar midpoint elevations but different distributions. Often the shape of the Cloud Radar condensation profile is more complicated, with multiple large peaks at different heights. Fair matches correspond to pairs of profiles with different midpoint elevations but similarly shaped distributions. Poor matches are events with different midpoint elevations and different 
TABLE 2. The average median heights grouped by method and comparison category. Excellent and good comparison categories tended to result from distributions with lower altitude medians. Fair and poor comparison categories tended to come from distributions with higher altitude medians. The Reanalysis method events categorized as poor matches have the highest average median height of all methods and categories.

\begin{tabular}{|c|c|c|c|c|}
\hline & Excellent & Good & Fair & Poor \\
\hline$M( \pm$ s.e.) Reanalysis method (MSL) & $1456(42.7)$ & $2141(46.1)$ & $3794(425)$ & $4782(182)$ \\
\hline$M$ (土s.e.) Cloud Radar method (MSL) & $1388(32.5)$ & $1894(50.2)$ & $2825(104)$ & $2557(159)$ \\
\hline
\end{tabular}

condensation profile shapes. Note that the examples with smaller $O$ tend to be profiles with substantially different shapes, which may be related to differences in $A$.

Because the reanalysis data reflect average meteorological conditions over $3 \mathrm{~h}$ in the $1^{\circ} \times 1^{\circ}$ pixel containing the ARM NSA station, as compared to the cloud radar data, which represent the 3-h-average cloud conditions at the ARM NSA station, no perfect agreement between the methods is expected. Nonetheless, it is useful to analyze the characteristics of the most similar and most different Cloud Radar method and Reanalysis method condensation profiles.

\section{3) EXCELLENT TO FAIR MATCHES BETWEEN METHODS}

Of the 68 events for which both methods produced a condensation profile, there were 28 excellent matches and 20 good matches. For these two categories, neither method produced a condensation profile with $M$ greater than $5000 \mathrm{~m}$. This, coupled with the information in Table 2, indicates that lower-elevation condensation profiles are more likely to match well, with the lowest-elevation profiles tending to match the best. The excellent matches tended to have very similar $A$ (not shown) and also produced some of the largest $A$ values for either method.

There were six events with a fair match between methods. These events were characterized by broader peaks with lower $A$ values for both the Reanalysis and Cloud Radar methods. No relationship was found between the quality of the match and whether the event was characterized as synoptic, regional, or local.

\section{4) Poor matches Between Methods}

There were 14 events that exhibited a poor match between methods. One of these (26 October 2009) was a local event characterized by heavy mixed-phase precipitation. The skew $T$ plot indicated layers of conditional instability within the lower troposphere between small temperature inversions. The Reanalysis profile produced high-elevation (7000-10000 MSL) clouds and very little condensation at lower elevations, perhaps an indication of GDAS capturing high-elevation ice clouds and stability in the lower atmosphere.
Ten of the 14 poor match events had positive $\Delta M$, corresponding to higher Reanalysis method $M$ relative to Cloud Radar method $M$. Seven of the 10 events had liquid precipitation and were characterized by large differences in amplitude $\Delta A$. The relationship between large $\Delta M$, large $\Delta A$, and precipitation type is shown in Fig. 2.

The relationship between large $\Delta M$ and liquid precipitation may be because liquid precipitation is more likely to attenuate the radar signal (Moran et al. 1998; Kollias et al. 2007), leading to a low bias in the Cloud Radar condensation profile. Higher-intensity precipitation of any type may also cause attenuation. But most moderate and heavy category precipitation did not exhibit large $\Delta M$ or large $\Delta A$ in our dataset. Another way the cloud radar may have contributed to the poor match was by missing small hydrometeors, like those in high-altitude ice clouds (Comstock et al. 2002). However, the value-added product ARSCL, which combines the best modes for sensing various precipitation types into one product, is designed to minimize this issue.

Only 4 of the 14 poor match events indicated negative $\Delta M$, corresponding to a lower Reanalysis method $M$ relative to the Cloud Radar method $M$. The skew $T$ plots (Media S2) indicate that these were events during which precipitation originated from moist layers in a relatively dry upper atmosphere, often above a temperature inversion. In two of the condensation profiles, the Reanalysis method produced substantial condensation in the very low atmosphere, which may indicate the presence of fog. For these outliers, the condensation profile generated by the Cloud Radar method is supported by the skew $T$ plots. Thus, for these storms the low bias of the Reanalysis method is either because the Reanalysis data did not resolve higher-elevation thin precipitating layers or because the prevailing conditions of the pixel reflected very low-elevation precipitation, which was in reality clouds or fog.

\section{5) Methodological SOURCES OF BIAS}

There are at least three general reasons why differences between the methods may occur.

First, as previously stated, the Cloud Radar method reflects the 3-h average conditions at a specific location in Barrow, whereas the Reanalysis method reflects the 


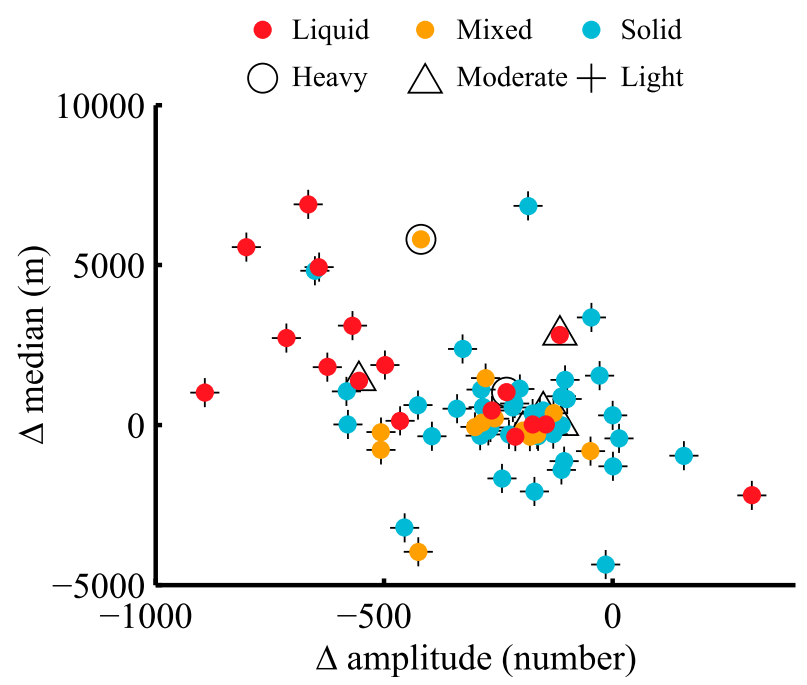

FIG. 2. Large differences in the methods' median values $\Delta M$ tend to occur with negative differences in normalized maximum amplitude $\Delta A$. Negative $\Delta A$ indicates the Cloud Radar method had higher $A$ than the Reanalysis method. For most of the strongly positive $\Delta M$ and strongly negative $\Delta A$, the precipitation type was liquid. Precipitation intensity did not strongly affect $\Delta M$, though most moderate- and high-intensity events have negative $\Delta A$.

3-h average conditions in the $1^{\circ} \times 1^{\circ}$ pixel containing the ARM NSA station. Comparing a point to a finite area will lead to inconsistencies in the resulting condensation profiles, particularly because the pixel containing Barrow is a mix of land, ocean, and, in some seasons, sea ice. The assumption that the Reanalysis data will accurately represent the meteorology at Barrow is likely not always true and may account for the majority of the error detected in our study.

Second, there may be error in the cloud radar measurements. The cloud radar returns have errors of $0.5 \mathrm{dBZ}$ for reflectivity and $0.1 \mathrm{~m} \mathrm{~s}^{-1}$ for Doppler vertical velocity. The MMCR and KAZR data quality reports indicate that the data are reliable for all but one event: the reflectivity measurement on 11 March 2013 exhibits a 4-5-dBZ increase. Zhao and Garrett (2008) estimate errors in the total amount of precipitation of up to $40 \%$ for ice and $15 \%$ for liquid precipitation at the $95 \%$ confidence level. However, we expect that the method produces reliable condensation rate profiles and that the uncertainty in condensation rate is constant with altitude.

Finally, the vertical structure of the cloud radar condensation profiles indicates that precipitation often occurs from thin clouds. This is consistent with the narrow layers of conditional instability sandwiched by stable layers observed in the skew $T$ plots, which likely result from weak updrafts, as documented by Shupe et al. (2008). These precipitation-producing moist, unstable layers are too narrow to be resolved by the vertical resolution of the reanalysis data. This issue is more notable at higher elevations because of the increasing height difference between pressure levels. Likewise, the method for condensation production used in the Reanalysis method is more similar to the typical model for groundheating-driven convection than it is to vertical motions induced by cloud-top radiative cooling, which are the typical driver of Arctic precipitation (Curry 1986).

\section{b. Vapor source location and conditions}

Back trajectory analysis has been used to interpret interevent precipitation isotope variation (e.g., Reijmer and Broeke 2001; Sodemann et al. 2008; Breitenbach et al. 2010; Theakstone 2011; Kurita 2013; Putman et al. 2017), with attention paid to the estimated source conditions. Mean source conditions for the trajectories are characterized by $\bar{V}_{\mathrm{SST}}, \bar{V}_{\mathrm{hSST}}$, and $\overline{\delta^{2} H_{v}}$ (Fig. 3) -all of which are relevant for isotope hydrology studies.

Among trajectories that traced similar paths, those that arrived at higher altitudes above Barrow usually originated at greater distance, typically corresponding to lower source latitude, warmer source sea surface temperatures, and ${ }^{2} \mathrm{H}$ enrichment in boundary layer vapor. As a result, events with $|\Delta M|$ less than $1500 \mathrm{~m}$, regardless of the value of $O$, tended to produce more similar $\bar{V}_{\text {SST }}$ and $\bar{\delta}^{2} H_{v}$. However, $\bar{V}_{\mathrm{hSST}}$ was similar across categories. The average characteristic values of each of the four categories (excellent to poor) are summarized in Table 3. The scatter present in Fig. 3 may be a result of vertical wind shear.

The greater $|\Delta M|$ categories (fair and poor), which typically result from greater Reanalysis method $M$ than Cloud Radar $M$, tended to estimate greater $\bar{V}_{\text {SST }}$ (up to $14^{\circ} \mathrm{C}$ ) and ${ }^{2} \mathrm{H}$ enrichment of boundary layer vapor (up to $17 \%$ ). This illustrates the importance of an accurate condensation rate profile for determining vapor source location. One may expect that approaches that do not take the condensation rate profile into account, such as using a limited number of air parcels or distributing parcels uniformly throughout the troposphere, could create even larger errors in reconstructed vapor source locations. On this basis, we recommend the Reanalysis method to researchers looking for a reliable method to map the source, yet remain computationally inexpensive compared to massively sampled back trajectories. However, the accuracy of the Reanalysis condensation profiles should be verified with radiosonde and other weather data when available.

\section{Conclusions}

In this work we describe and test a novel method for estimating the distribution of altitudes at which 


$$
\begin{aligned}
& \bullet-\Delta_{\mathrm{M}}|<1500 \mathrm{~m}, \mathrm{O}>0.75 \cdots| \Delta \mathrm{M} \mid>1500 \mathrm{~m}, \mathrm{O}>0.75 \\
& \cdots|\Delta \mathrm{M}|<1500 \mathrm{~m}, \mathrm{O}<0.75 \cdots|\Delta \mathrm{M}|>1500 \mathrm{~m}, \mathrm{O}<0.75
\end{aligned}
$$
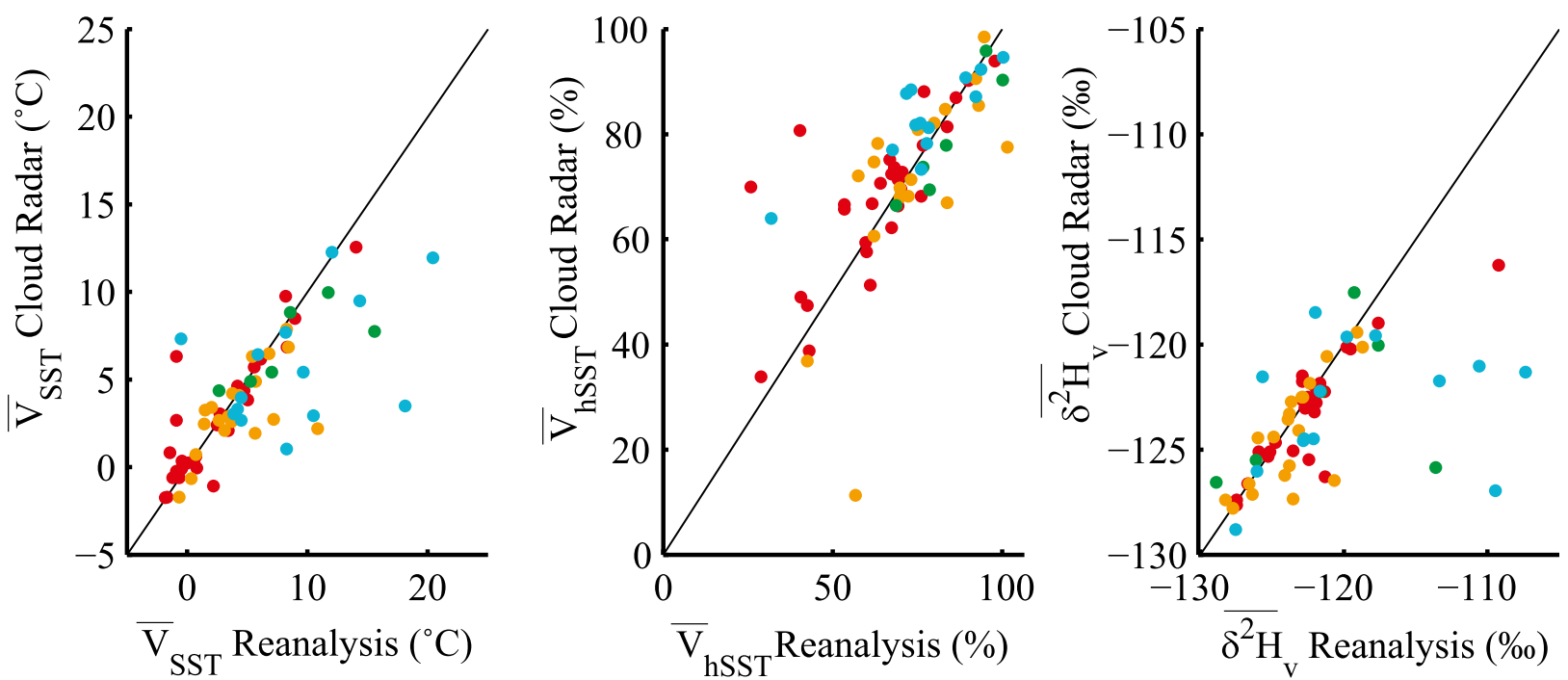

FIG. 3. Differences between the two methods in light of their use in precipitation stable isotope systematics studies can be characterized by comparing the average estimated vapor source conditions. The three metrics chosen $\bar{V}_{\mathrm{SST}}, \bar{V}_{\mathrm{hSST}}$, and $\bar{\delta}^{2} H_{v}$. Vapor source characteristics tend to be the most similar for the excellent matches (red) and most dissimilar for the poor matches (blue) for $\bar{V}_{\text {SST }}$ and $\bar{\delta}^{2} H_{v}$. However, for $\bar{V}_{\mathrm{hSST}}$, the category is not related to the magnitude of difference between the characteristic value estimated by the two methods.

precipitation originates as input for a Lagrangian back trajectory program used to identify vapor source regions. We emphasize that locating the proper altitude range for back trajectory initiation is important for accurate identification of the source region because wind direction and speed vary with altitude. We also recommend that a condensation rate profile be determined so that each air parcel traced with a back trajectory represents an equal fraction of the total precipitation produced.

In this paper we introduce the Reanalysis method for determining a condensation rate profile. The Reanalysis method uses globally available reanalysis meteorological data and an air lofting model. We compare the Reanalysis method with the Cloud Radar method, which uses direct high-resolution cloud measurements. The two methods are not equivalent because the Cloud Radar is a point observation, while Reanalysis data are an average across a $1^{\circ} \times 1^{\circ}$ pixel containing land, ocean, and sometimes sea ice. The analysis shows the circumstances under which the methods are comparable, which informs the applicability of the Reanalysis method at other locations. The condensation profiles produced by each method are compared using the median $M$, median-adjusted overlap $O$, and amplitude $A$.

For events for which condensation rate profiles were produced (68 of 70 events), the matches were categorized as excellent $(n=28)$, good $(n=20)$, fair $(n=6)$, and poor $(n=14)$, based on $|\Delta M|$ and $O$. Contrary to expectation, event categorization into local, regional, and synoptic groups was not successful at predicting how well the methods would compare. Instead, excellent matches tended to have lower $M$, large Cloud Radar $A$, and small $\Delta A$. Excellent and good category events produced similar estimates of vapor source conditions,

TABLE 3. How the quality of match between condensation profiles affects the estimated vapor source conditions. Matches in the fair and poor categories tend to exhibit greater differences between the estimated conditions at the source relative to excellent and good cate-

\begin{tabular}{|c|c|c|c|c|}
\hline & Excellent & Good & Fair & Poor \\
\hline$\Delta \bar{V}_{\text {SST }}( \pm$ s.e. $){ }^{\circ} \mathrm{C}$ & $-0.2629(0.0660)$ & $0.9723(0.1173)$ & $1.5986(0.5479)$ & $3.0814(0.3810)$ \\
\hline$\Delta \bar{V}_{\text {hSST }}( \pm$ s.e. $) \%$ & $-4.89(0.43)$ & $1.95(0.70)$ & $4.85(0.69)$ & $-6.41(0.74)$ \\
\hline$\Delta \bar{\delta}^{2} H_{v}( \pm$ s.e. $) \%$ & $0.7047(0.0620)$ & $0.5316(0.0887)$ & $1.7824(0.9007)$ & $3.7001(0.4584)$ \\
\hline
\end{tabular}
gories. However, the relative humidity with respect to $\Delta \bar{V}_{\mathrm{hSST}}$ did not exhibit substantial differences among the categories. 
indicating that the median height of the back trajectory initialization was a more important predictor of the vapor source location than the shape of the condensation profile.

Poor matches between the methods were typically characterized by large, positive $\Delta M$, corresponding to a higher median for the Reanalysis method condensation profile relative to the Cloud Radar method. Many of these events were characterized by liquid precipitation from thin low-elevation clouds. The large positive bias of the Reanalysis method may be due to attenuation of the radar, precipitation localized to Barrow, or lower-resolution reanalysis data not representing thin cloud layers.

Within our study, low- and medium-elevation clouds over Barrow were typically well represented by the Reanalysis method. Based on our comparison, the Reanalysis method is able to represent a point within a pixel when precipitation results from cloud layers thick enough to be resolved by the Reanalysis and the average conditions of the pixel are conducive to precipitation. The method tends to be less accurate with complex temperature and humidity profiles. This method should be used with caution when the meteorology indicates precipitation from intense convection, or very localized conditions. For studies of vapor source characteristics, this method will produce accurate and spatially robust results. It is an improvement over methods that track a small number of air parcels from fixed heights, yet it remains relatively computationally inexpensive.

Acknowledgments. Data appearing in all figures of this paper are included in the supplement, Table S1. The files containing the raw and processed along-trajectory data from which the presented data were calculated are available upon request by contacting Annie Putman (putmanannie@gmail.com).The MATLAB subroutines pertaining to the specific methods are available in the supplement. The other MATLAB codes used to perform this analysis are available upon request from Annie Putman. The cloud radar and radiosonde data referenced in the manuscript are available from the Atmospheric Radiation Monitoring archive (at https://www. arm.gov/data). The Lagrangian back trajectory program, HYSPLIT, was downloaded from NOAA's Air Resources Laboratory website (http://ready.arl.noaa. gov/HYSPLIT.php). The HYSPLIT GUI was used to download GDAS reanalysis files.

This project was supported by NSF Grant 1022032. The authors thank Walter Brower and Jimmy Ivanhoff for their sample collection efforts at the ARM NSA station. The authors appreciate the valuable comments of Ben Kopec and two anonymous reviewers.

\section{REFERENCES}

Alduchov, O. A., and R. E. Eskridge, 1996: Improved Magnus form approximation of saturation vapor pressure. J. Appl. Meteor., 35, 601-609, doi:10.1175/1520-0450(1996)035<0601: IMFAOS $>2.0 . \mathrm{CO} ; 2$.

Bailey, H. L., D. S. Kaufman, A. C. G. Henderson, and M. J. Leng, 2015: Synoptic scale controls on the $\delta 18 \mathrm{O}$ in precipitation across Beringia. Geophys. Res. Lett., 42, 4608-4616, doi:10.1002/2015GL063983.

Bharadwaj, N., D. Nelson, I. Lindenmaier, and K. Johnson, 2011: Ka ARM zenith radar (KAZRGE). Atmospheric Radiation Measurement Climate Research Facility Data Archive. Subset used: KAZRGE 1 April 2011-1 April 2013, 71.323N, 156.609 ${ }^{\circ} \mathrm{W}$ : North Slope Alaska Central Facility, Barrow, AK (C1), accessed 1 May 2013, doi:10.5439/1025214.

Breitenbach, S. F. M., J. Adkins, H. Meyer, N. Marwan, K. K. Kumar, and G. H. Haug, 2010: Strong influence of water vapor source dynamics on stable isotopes in precipitation observed in Southern Meghalaya, NE India. Earth Planet. Sci. Lett., 292, 212-220, doi:10.1016/j.eps1.2010.01.038.

Clothiaux, E. E., T. P. Ackerman, G. G. Mace, K. P. Moran, R. T. Marchand, M. A. Miller, and B. E. Martner, 2000: Objective determination of cloud heights and radar reflectivities using a combination of active remote sensors at the ARM CART sites. J. Appl. Meteor., 39, 645-665, doi:10.1175/ 1520-0450(2000)039<0645:ODOCHA > 2.0.CO;2.

Comstock, J., T. Ackerman, and G. Mace, 2002: Ground-based lidar and radar remote sensing of tropical cirrus clouds at Nauru Island: Cloud statistics and radiative impacts. J. Geophys. Res., 107, 4714, doi:10.1029/2002JD002203.

Curry, J. A., 1986: Interactions among turbulence, radiation and microphysics in Arctic stratus clouds. J. Atmos. Sci., 43, 90-106, doi:10.1175/1520-0469(1986)043<0090: IATRAM > 2.0.CO;2.

Draxler, R. R., 1999: HYSPLIT_4 user's guide. NOAA Tech. Memo. ERL ARL-230, 35 pp., http://www.arl.noaa.gov/ documents/reports/arl-230.pdf.

— eling system. NOAA Tech. Memo. ERL ARL-224, 27 pp.

— system of trajectories, dispersion, and deposition. Aust. Meteor. Mag., 47, 295-308.

Everhart, K. K., 2011: Sea ice and the Arctic water cycle: A novel application of stable isotope meteorology in Barrow and Atqasuk, Alaska. M.S. thesis, Dept. of Earth Science, Dartmouth College, 105 pp., https://search.proquest.com/docview/921650191.

Gimeno, L., and Coauthors, 2012: Oceanic and terrestrial sources of continental precipitation. Rev. Geophys., 50, RG4003, doi:10.1029/2012RG000389.

Good, S., D. Noone, N. Kurita, M. Benetti, and G. Bowen, 2015: $\mathrm{D} / \mathrm{H}$ isotope ratios in the global hydrologic cycle. Geophys. Res. Lett., 42, 5042-5050, doi:10.1002/2015GL064117.

Illingworth, A. J., and Coauthors, 2007: Cloudnet: Continuous evaluation of cloud profiles in seven operational models using ground-based observations. Bull. Amer. Meteor. Soc., 88, 883-898, doi:10.1175/BAMS-88-6-883.

Johnson, K., and M. Jensen, 1996: Active remotely-sensed cloud locations. Atmospheric Radiation Measurement Climate Research Facility. Subset used: ARSCL1CLOTH 1 January 2009-1 April 2013, $71.323^{\circ} \mathrm{N}, 156.609^{\circ} \mathrm{W}$ : North Slope Alaska (NSA) Central Facility, Barrow AK (C1), accessed 1 January 2013. 
Keegan, K. M., M. R. Albert, J. R. McConnell, and I. Baker, 2014: Climate change and forest fires synergistically drive widespread melt events of the Greenland Ice Sheet. Proc. Natl. Acad. Sci. USA, 111, 7964-7967, doi:10.1073/pnas.1405397111.

Kollias, P., E. E. Clothiaux, M. Miller, B. Albrecht, G. Stephens, and T. Ackerman, 2007: Millimeter-wavelength radars: New frontiers in atmospheric cloud and precipitation research. Bull. Amer. Meteor. Soc., 88, 1608-1624, doi:10.1175/BAMS-88-10-1608.

Kurita, N., 2013: Origin of Arctic water vapor during the icegrowth season. Geophys. Res. Lett., 38, L02709, doi:10.1029/ 2010 GL046064.

Moran, K., B. Martner, M. Post, R. Kropfli, D. Welsh, and K. Widener, 1998: An unattended cloud-profiling radar for use in climate research. Bull. Amer. Meteor. Soc., 79, 443-455, doi:10.1175/1520-0477(1998)079<0443:AUCPRF>2.0.CO;2.

NOAA, 2013: NOAA optimum interpolation (OI) sea surface temperature (SST) V2. ESRL PSD, accessed 1 March 2013, https://www.esrl.noaa.gov/psd/data/gridded/data.noaa.oisst. v2.html.

Nusbaumer, J., T. E. Wong, C. Bardeen, and D. Noone, 2017: Evaluating hydrological processes in the Community Atmosphere Model Version 5 (CAM5) using stable isotope ratios of water. J. Adv. Model. Earth Syst., 9, 949-977, doi:10.1002/ 2016MS000839.

O'Gorman, P. A., and T. Schneider, 2009: The physical basis for increases in precipitation extremes in simulations of 21stcentury climate change. Proc. Natl. Acad. Sci. USA, 106, 14 773-14777, doi:10.1073/pnas.0907610106.

Pfahl, S., and H. Wernli, 2008: Air parcel trajectory analysis of stable isotopes in water vapor in the eastern Mediterranean. J. Geophys. Res., 113, D20104, doi:10.1029/2008JD009839.

Putman, A. L., X. Feng, L. Sonder, and E. Posmentier, 2017: Annual variation in event-scale precipitation $\delta^{2} \mathrm{H}$ at Barrow, $\mathrm{AK}$, reflects vapor source region. Atmos. Chem. Phys., 17, 46274639, doi:10.5194/acp-17-4627-2017.

Reijmer, C. H., and M. R. V. D. Broeke, 2001: Moisture source of precipitation in Western Dronning Maud Land, Antarctica. Antarct. Sci., 13, 210-220, doi:10.1017/S0954102001000293.

Shupe, M. D., P. Kollias, P. O. G. Persson, and G. M. McFarquhar, 2008: Vertical motions in Arctic mixed-phase stratiform clouds. J. Atmos. Sci., 65, 1304-1322, doi:10.1175/2007JAS2479.1.

Sodemann, H., C. Schwierz, and H. Wernli, 2008: Interannual variability of Greenland winter precipitation sources: Lagrangian moisture diagnostic and North Atlantic Oscillation influence. J. Geophys. Res., 113, D03107, doi:10.1029/2007JD008503.

Stohl, A., and P. James, 2004: Lagrangian analysis of the atmospheric branch of the global water cycle. Part I: Method description, validation, and demonstration for the August 2002 flooding in central Europe. J. Hydrometeor., 5, 656-678, doi:10.1175/1525-7541(2004)005<0656:ALAOTA>2.0.CO;2.

— branch of the global water cycle. Part II: Moisture transports between earth's ocean basins and river catchments. J. Hydrometeor., 6, 961-984, doi:10.1175/JHM470.1.

Theakstone, W. H., 2011: A seven-year study of oxygen isotopes in daily precipitation at a site close to the Arctic Circle, Tustervatn, Norway: Trajectory analysis and links with the North Atlantic Oscillation. Atmos. Environ., 45, 5101-5109, doi:10.1016/j.atmosenv.2011.06.034.

Wernli, H., M. Paulat, M. Hagen, and C. Frei, 2008: SAL-A novel quality measure for the verification of quantitative precipitation forecasts. Mon. Wea. Rev., 136, 4470-4487, doi:10.1175/2008MWR2415.1.

Zhao, C., and T. J. Garrett, 2008: Ground-based remote sensing of precipitation in the Arctic. J. Geophys. Res., 115, D14204, doi:10.1029/2007JD009222. 